\title{
Optimización de un Sistema de Abastecimiento de Pintura a Concesionarios de Baja y Media Demanda
}

Paul Taboada-González(1), Quetzalli Aguilar-Virgen (1), Jose Ibarra-Trujillo(1) y María Ramírez-Barreto(2) (1) Facultad de Ciencias Químicas e Ingeniería, Univ. Autónoma de Baja California. Calzada Universidad No. 14418, Mesa de Otay, C.P. 22390, Tijuana, Baja California, México (e-mail: ptaboada@uabc.edu.mx) (2) Instituto de Ingeniería, Universidad Autónoma de Baja California. Calle de la Normal S/N y Blvd. Benito Juárez, Col. Insurgentes Este, C.P. 21100, Mexicali, Baja California, México.

Recibido Oct. 30, 2015; Aceptado Ene. 6, 2016; Versión final Ene. 18, 2016, Publicado Jun. 2016

\begin{abstract}
Resumen
El objetivo de este estudio fue optimizar el sistema de administración de inventarios en un centro de distribución que ayude a incrementar la productividad de la empresa. Esto es de interés porque uno de los aspectos de éxito de una empresa se refiere a la adecuada operación de los centros de distribución. Se hizo una clasificación $A B C$ de los artículos más representativos de una empresa de distribución de pinturas y se realizaron proyecciones empleando el software Oracle CrystalBall ${ }^{\circledR}$. Se realizó un estudio de las instalaciones y equipo de manejo de materiales. Los porcentajes de utilización anual son $47.87 \%$ para los artículos clase A, 38.24\% para los artículos clase B y 13.89 para los artículos clase C. La capacidad actual de almacenaje es suficiente para la demanda. El trabajo muestra que el desarrollo de políticas de inventario adecuadas permite mantener un resurtido dinámico al centro distribución.
\end{abstract}

\section{Optimization of a Paint Supply System to Small and Moderate Demand Concessioners}

\begin{abstract}
The aim of this study was to optimize a system for inventory management in a distribution center to help increasing business productivity. This is of interest because one aspect for the success of a company is related to the proper operation of the distribution centers. An ABC classification of the most representative items of a paint distribution company was carried out, and projections were done using the Oracle software CrystalBall ${ }^{\circledR}$. A study of the facilities and material handling equipment was performed. The annual utilization rates are $47.87 \%$ for the class A items, $38.24 \%$ for Class B items and $13.89 \%$ to class $C$ items. The current storage capacity is sufficient for the present demand. This work shows that the development of appropriate inventory policies keeps a dynamic refill in the distribution center.
\end{abstract}




\section{INTRODUCCIÓN}

La entrega de los bienes con la mayor eficacia es fundamental para la supervivencia de una empresa. Es primordial coordinar eficientemente el control del trabajo en proceso, el flujo de materiales y productos -así como los servicios de embarque desde el punto de origen hasta el punto de venta- para entregar al cliente el producto en tiempo y forma, con la calidad requerida. Wu et al., (2015) indican que muchas empresas consideran el tiempo de entrega como una estrategia importante y se esfuerzan por acortarlo lo más posible para mejorar la eficiencia del inventario.

Es inevitable que los perfiles de demanda cambien con el tiempo debido a la competencia, la introducción de nuevos productos, la madurez del producto, y la estacionalidad, entre otros (Carlo y Giraldo, 2012). Esta situación puede afectar la disponibilidad de productos si no existe una adecuada política de inventarios. Andrade et al., (2014) indican que la mejor forma de cumplir con los tiempos de entrega del producto y evitar futuros costos, es monitorear los pedidos de los clientes y tener productos en exceso como una protección. Sin embargo, un exceso de inventario puede ser riesgoso debido a que existen costos asociados (producción, traslado, almacenaje, protección del inventario, mermas, obsolescencia, impuestos entre otros). Pan et al., (2015) indican que en el sector de productos de gran consumo, los costos de inventario generalmente representan hasta el $40 \%$ del total de los costos logísticos, por no mencionar el costo de la escasez en las tiendas al por menor, de aproximadamente el $7 \%$ de los productos en el supermercado. Por tanto, determinar eficazmente las políticas de existencias de artículos contribuye directamente en la competitividad de una empresa.

Una vez que los productos están en el almacén, su distribución influye en el tiempo de preparación y despacho de pedidos. Si la distribución es inadecuada, se incrementan los recorridos, se crean espacios muertos y se elevan los tiempos de preparación. Carlo y Giraldo (2012) indican que la distribución eventualmente se convierte en ineficiente debido a los cambios de la demanda, por lo que es necesario reorganizarla. Pazour y Carlo, (2015) indican que la reorganización se completa habitualmente en el lanzamiento de un nuevo producto, al final de cada temporada, o sobre una base mensual, semanal o incluso diariamente, dependiendo de la volatilidad del perfil de la demanda. En consecuencia, el diseño de las políticas de reorganización que reduzcan al mínimo los recorridos es un componente importante de las operaciones de almacén eficaces.

La operación del almacén Tijuana de la empresa Greenpaint (el nombre real se omite por confidencialidad) se está viendo afectada debido a la diversificación de su mercado. Desde sus inicios, el almacén estaba dedicado al área profesional-industrial y reproceso de producto terminado. No obstante, recientemente se incorporó a la cartera de productos la pintura vinílica. Esta nueva actividad ha generado nuevos retos a la administración debido a pérdidas económicas por ventas fallidas y limitantes operativas que están afectando a los concesionarios. Entre los aspectos que han influido en la nueva problemática se encuentran el retraso en mercancías por imprevistos, el costo de flete generado por el volumen de pedidos y/o tonelaje que solicitan los concesionarios (detallistas), y los tiempos de envíos generados por la consolidación de cargas por parte del transportista.

La venta de pintura vinílica representa un ingreso importante para la empresa, por lo que una mejora en su administración incrementaría la rentabilidad de la empresa. Li et al., (2015) indican que el sistema de administración de inventarios tiene un impacto considerable en el desempeño de los centros de distribución y de la totalidad de la red de suministros. Por su parte, Gutierrez y Vidal (2008) indican que para que las empresas mantengan su competitividad, es necesario definir una metodología que permita estimar las políticas de control de inventarios de productos. Por tanto, la eficiencia y capacidad de los centros de distribución dependen grandemente de la asignación de almacenamiento y de la preparación de pedidos.

Desde la óptica estratégica, se reconoce la necesidad de mejorar en al menos cinco prioridades competitivas: costo, calidad, entregas, flexibilidad y servicio (Trujillo et al., 2015). Bajo esta perspectiva, un nuevo plan de acción que considere la pintura vinílica que consumen los concesionarios permitirá reducir los costos al cliente final, incrementar la calidad en el servicio, mejorar las entregas, elevar la flexibilidad en los productos y elevar el servicio hacia los concesionarios. Por lo expuesto anteriormente, el objetivo de este estudio fue optimizar el sistema de administración de inventarios para aprovechar espacios, recibir más producto y mejorar el servicio a los concesionarios e incrementar la productividad de la empresa.

\section{METODOLOGÍA}

Se realizó una lluvia de ideas (brainstorming) y se estratificaron las propuestas con las categorías relevantes. Con esta información se construyó un diagrama Causa-Efecto para identificar los elementos que influyen en la operación del almacén. Posteriormente, se analizaron las categorías y se definieron los aspectos operativos que pudieran ser modificables. Una vez concluido este proceso, se inició el estudio en las áreas de oportunidad. En este proceso participaron directivos de la empresa y los autores. 


\section{Definición de productos y cantidades}

Para realizar las proyecciones de venta, se determinaron los artículos más representativos y se realizó una clasificación. El análisis $A B C$ recientemente ha reconocido la escasez, el costo de inventario unidad de retención, la reparabilidad, requisito de tamaño pedido, como criterios importantes que afectan a la clasificación de inventario. No obstante, el criterio que se emplea con mayor frecuencia el valor de la demanda anual de este artículo (Hatefi y Torabi, 2015). En este estudio la clasificación ABC se realizó considerando el grado de ventas y el costo para el cliente final. En este último se consideraron productos que, a pesar de que no se venden a grandes volúmenes en la región, representarían un alto costo para el cliente si se surtieran desde la planta de producción hasta el momento de su consumo.

\section{Proyección de las ventas}

Se recabo información de históricos de ventas para realizar la proyección de ventas. Por medio de paquetes tecnológicos de información y bases de datos ORACLE ${ }^{\circledR}$ de la empresa, se encontró la información de ventas diaria, faltantes de productos, niveles de inventarios, entre otros. Se tomó como punto de partida el histórico de ventas de un año, considerando suficiente este periodo de tiempo para desarrollar las proyecciones de ventas. Para lograr una buena precisión en las proyecciones, se realizaron estimaciones por diversos métodos y se hicieron comparaciones entre ellos para seleccionar aquel que presente un menor error. Se aplicaron métodos no estacionales (Promedio móvil simple, Suavizado exponencial simple, Promedio móvil doble, Suavizado exponencial doble), métodos estacionales (Aditivo estacional, Multiplicativo estacional, Aditivo de Holt-Winters, Multiplicativo de Holt-Winters) y ARIMA (Autoregressive Integrated Moving Average). Sobre éste último, Sarpong (2013) indica que los modelos ARIMA son especialmente adecuados para la predicción a corto plazo, debido a que el modelo pone más énfasis en el pasado reciente en lugar de un pasado lejano. Las previsiones a largo plazo de los modelos ARIMA son menos fiables que las previsiones a corto plazo.

Para la obtención de los pronósticos se consideró como criterio de selección el menor valor de Error Porcentual Absoluto Medio (MAPE, Mean Absolute Percentage Error) de los distintos métodos para elegir. Con ello se buscó reducir la incertidumbre la proyección de venta de los distintos productos. EI MAPE (expresado como porcentaje) relaciona el error de pronóstico con el nivel de la demanda, y es útil para colocar el rendimiento del pronóstico en su correcta perspectiva. Para estimar su valor se emplea la ecuación 1.

$$
\text { MAPE }=\frac{\sum\left[\left|e_{p}\right|(100)\right] / \text { demandareal }}{n}
$$

Donde $e_{p}$ representa el error del pronóstico y $n$ es el número total de periodos. En este estudio, el MAPE se estimó usando el software Oracle CrystalBal ${ }^{\circledR}$ en cada artículo considerado. Al final se realizó un concentrado para analizar las proyecciones.

\section{Estudio de las instalaciones y equipo de manejo de materiales}

Se desarrolló una distribución de planta actual y se realizaron bosquejos de la instalación. Con esta información se realizó un plano que se empleó para una nueva verificación y tomar medidas más precisas. Se recolectó información sobre las características de los estantes (altura, profundidad, longitud, capacidad de carga), las dimensiones de los pasillos y las características del equipo para el manejo de materiales (montacargas).

\section{Estimación de espacio de almacenaje}

Para transportar y almacenar las cubetas -que son las que presentan mayor peso por unidad de embalaje- se cuenta con dos presentaciones: Tarima (pallet) de 48 (T-48) y Tarima de 36 (T-36). El nombre del embalaje se deriva del número de cubetas colocadas en la tarima. Así, la T-48 se conforma por 4 cubetas de ancho, 3 cubetas de largo y 4 estibas $(4 \times 3 \times 4)$ mientras que la T-36 tiene un arreglo3 $\times 4 \times 3$. Para estimar la cantidad de $\mathrm{m}^{3}$ requeridos, se dividieron las cantidades vendidas de cada producto en cada mes entre la cantidad de piezas que se estiban en una T-48. Se consideró la T-48 para efectos de cálculo debido a que es el arreglo con el que más se aprovecha el espacio cúbico de los estantes.

\section{Determinación de niveles de inventarios}

Se estimaron las cantidades máxima, mínima y punto de pedido de los productos de los Artículos ABC para controlar el inventario. Los niveles se obtuvieron empleando las ecuaciones 2,3 y 4. 


$$
\begin{aligned}
& P_{r}=\left(E_{s}\right)\left(D_{d}\right)+\left(T_{e}\right)\left(D_{d}\right) \\
& C_{m}=\left(E_{s}\right)+\left(C_{p}\right) \\
& C_{p a}=1 / 2\left(C_{p}\right)+\left(E_{s}\right)
\end{aligned}
$$

Donde $\mathrm{P}_{\mathrm{r}}=$ punto pedido de re-orden; $\mathrm{E}_{\mathrm{s}}=$ Existencia de seguridad en días; $\mathrm{T}_{\mathrm{e}}=$ tiempo de entrega en días; $\mathrm{D}_{\mathrm{d}}=$ demanda por días; $\mathrm{C}_{\mathrm{m}}=$ cantidad máxima a almacenar; $\mathrm{C}_{\mathrm{p}}=$ cantidad del pedido; $\mathrm{C}_{\mathrm{pa}}=$ cantidad promedio que se va a almacenar. Este método para determinar las cantidades máximas y mínimas ha sido expuesto por diversos autores (Murthy y Gurusamy, 2009; Periasamy, 2009).

\section{RESULTADOS Y DISCUSIÓN}

El análisis dio como resultado la cantidad de 63 artículos que se deben de tener en inventario para cubrir la demanda en esta zona. La clasificación ABC se conformó de la siguiente manera: 8 artículos clase A (12.7\%), 20 artículos clase B (31.75\%) y 35 artículos clase C (55.55\%). Los porcentajes de utilización anual son $47.87 \%$ para los artículos clase A, 38.24\% para los artículos clase B y 13.89 para los artículos clase C.

\section{Proyección de las ventas}

Los datos totales de las ventas para el año de análisis se presentan en la Tabla 1.

Tabla 1: Concentrado de ventas para el año de análisis

\begin{tabular}{|l|c|c|c|c|c|c|c|c|c|c|c|c|}
\hline Descripción & Ene & Feb & Mar & Abr & May & Jun & Jul & Ago & Sep & Oct & Nov & Dic \\
\hline Cubetas & 4,522 & 3382 & 4465 & 4679 & 2774 & 4706 & 5738 & 4937 & 2467 & 4871 & 12084 & 6153 \\
\hline Galones & 1,216 & 1,228 & 1,184 & 1,792 & 1,028 & 1,632 & 2,292 & 1,512 & 1,220 & 1,988 & 3,256 & 2,588 \\
\hline Litros & 42 & 6 & 30 & 6 & 18 & 12 & 18 & 6 & 30 & 30 & 12 & 42 \\
\hline
\end{tabular}

En las tablas 2, 3 y 4 se presentan las proyecciones para los artículos que tienen un MAPE menor de $20 \%$ de error, con la finalidad de presentar un pronóstico más preciso. Dicho porcentaje concuerda por lo expuesto por Kumar y Vanajakshi (2015), que indican que cualquier pronóstico con un valor MAPE de menos de 10\% puede ser considerado altamente preciso, $11-20 \%$ es bueno, 21 a $50 \%$ es razonable y $51 \%$ o más es incorrecto.

En la Tabla 2 se muestra las proyecciones con una confiabilidad del 95\% de seis meses de tres artículos considerados como tipo A. Se obtuvo un MAPE de 15.76\% para el artículo XJ3-200335, de 7.90\% para el XJ3-200333 y de 17.01\% para el XJ3-200804. El método de mejor proyección fue el SARIMA de un total de nueve métodos (cuatro métodos no estacionales, cuatro métodos estacionales y el método del promedio móvil autoregresivo integrado). Las proyecciones obtuvieron un coeficiente Durbin-Watson del 2 al 2.5 indicando que no existe autocorrelación y que los residuos son completamente independientes. Por tanto, se cumple con los criterios de calidad del modelo.

Tabla 2: Proyección mensual de artículos clase A

\begin{tabular}{|c|r|c|r|}
\hline \multirow{2}{*}{ Mes } & \multicolumn{3}{|c|}{ Ítems clasificados como A } \\
\cline { 2 - 4 } & XJ3-200335 & XJ3-200333 & XJ3-200804 \\
\hline Ene & $352 \pm 192$ & $692 \pm 78$ & $28 \pm 173$ \\
\hline Feb & $154 \pm 298$ & $610 \pm 78$ & $789 \pm 191$ \\
\hline Mar & $380 \pm 307$ & $452 \pm 83$ & $622 \pm 224$ \\
\hline Abr & $1,386 \pm 317$ & $533 \pm 96$ & $0 \pm 97$ \\
\hline May & $2,111 \pm 320$ & $46 \pm 104$ & $834 \pm 292$ \\
\hline Jun & $1,328 \pm 344$ & $206 \pm 104$ & $1,138 \pm 295$ \\
\hline
\end{tabular}

En la figura 1 se muestra la proyección para un artículo que tiene una clasificación A. Se observa que el modelo se ajusta adecuadamente a los datos históricos, por lo que su porcentaje de error es relativamente bajo. Esto se observa a través del MAPE, con un $15.76 \%$. El método que se utilizó como mejor opción es el SARIMA $(2,1,2)(1,0,1)$, con un coeficiente Durbin-Watson de 2.09 . 


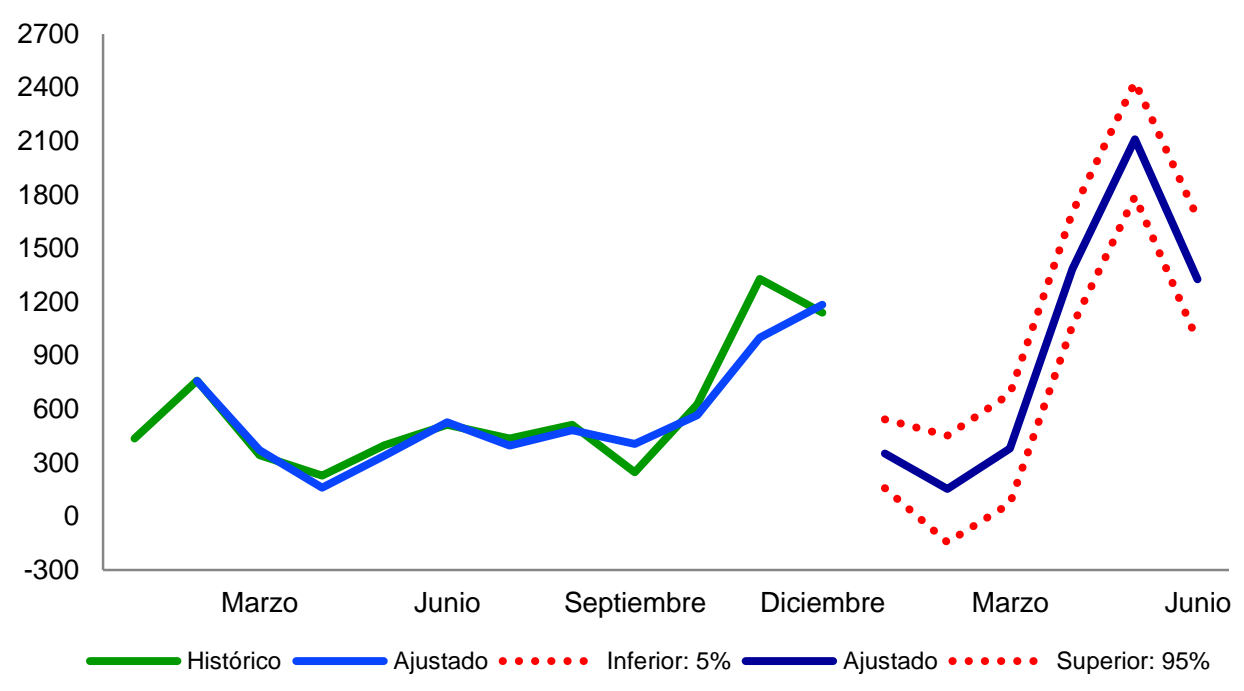

Fig. 1: Proyección de un artículo clase A

En la Tabla 3 se muestran las proyecciones de seis meses de los artículos considerados como tipo B, con una confiabilidad del $95 \%$. En los seis artículos se tiene que el coeficiente de Durbin-Watson oscila entre 1.68 y 2.05, lo que se considera como residuos independientes por estar en el rango de 1.5 y 2.5. Los métodos empleados fueron SARIMA con un MAPE que oscila entre $10.15 \%$ y $13.88 \%$, exceptuando el artículo XJ3400804 con un $18.29 \%$.

Tabla 3: Proyección mensual de artículos clase B

\begin{tabular}{|c|c|c|c|c|c|c|}
\hline \multirow{2}{*}{ Mes } & \multicolumn{7}{|c|}{ Ítems clasificados como B } \\
\cline { 2 - 7 } & XJ3-400335 & XJ3-200802 & XJ3-400805 & XJ3-408100 & XJ3-400804 & XJ3-400334 \\
\hline Ene & $239 \pm 47$ & $343 \pm 41$ & $141 \pm 32$ & $72 \pm 30$ & $179 \pm 27$ & $76 \pm 15$ \\
\hline Feb & $225 \pm 48$ & $127 \pm 59$ & $252 \pm 41$ & $93 \pm 36$ & $150 \pm 30$ & $53 \pm 15$ \\
\hline Mar & $341 \pm 54$ & $18 \pm 62$ & $78 \pm 43$ & $167 \pm 36$ & $106 \pm 32$ & $93 \pm 16$ \\
\hline Abr & $283 \pm 55$ & $0 \pm 53$ & $110 \pm 46$ & $152 \pm 36$ & $194 \pm 34$ & $68 \pm 18$ \\
\hline May & $342 \pm 71$ & $0 \pm 0$ & $223 \pm 51$ & $34 \pm 36$ & $164 \pm 36$ & $43 \pm 18$ \\
\hline Jun & $356 \pm 73$ & $100 \pm 83$ & $151 \pm 51$ & $49 \pm 36$ & $252 \pm 37$ & $62 \pm 19$ \\
\hline
\end{tabular}

En la Tabla 4 se exponen los pronósticos de los artículos clasificados como $\mathrm{C}$ que tuvieron un error menor al $20 \%$, con una confiabilidad el $95 \%$. El método que prevaleció para todos los artículos con una mejor proyección fue el SARIMA. Los porcentajes de MAPE en siete de los 12 artículos van del $10.29 \%$ al $14.97 \%$ y los cinco artículos restantes oscilan alrededor del $18.07 \%$ con una desviación estándar del $1 \%$. En estos artículos se tiene que el coeficiente de Durbin-Watson se encuentra en un rango del 1.51 al 2.30.

\section{Estudio de las instalaciones y equipo de manejo de materiales}

Se identificaron las características de las tarimas y productos que se colocarán en los estantes metálicos. Las dimensiones de los productos y los embalajes empleados para su transporte, se indican en la Tabla 5.

El área destinada para el almacenaje es de $340 \mathrm{~m}^{2}$. Para el almacenaje se cuenta con 8 estantes metálicos de tres niveles cada uno. Los estantes tienen una dimensión de 165"x89"x36" (420x226x91cm, altura, frente y fondo). La capacidad de carga de cada estante es de $6000 \mathrm{~kg}$, divididas en tres niveles. Los niveles tienen una capacidad de almacenaje de $2.55 \mathrm{~m}^{3}$ por nivel y cada uno admite 2 tarimas. En total se tiene una capacidad de 6 tarimas por estante y de 48 tarimas en total. Para el movimiento de cargas, se cuenta con un montacargas eléctrico Nissan M02 con capacidad de carga de 5,460 kg. Se emplea un montacargas eléctrico debido a que es un espacio cerrado y no se debe usar montacargas de combustión a gasolina o gas en lugares cerrados debido a la generación de monóxido de carbono, el cual es altamente venenoso. Téllez et al., (2006) indican que el estándar de calidad de aire para monóxido de carbono fijado por la Organización Mundial de la Salud es de 35 partes por millón por hora. 
Tabla 4: Proyección mensual de artículos clase C

\begin{tabular}{|c|c|c|c|c|c|c|}
\hline \multirow{2}{*}{ Mes } & \multicolumn{6}{|c|}{ Ítems clasificados como C } \\
\hline & XJ3-208106 & XJ3-400918 & XJ3-200801 & XJ3-201234 & XJ3-201132 & XJ3-200902 \\
\hline Ene & 0 & $51 \pm 17$ & $59 \pm 95$ & 0 & $0 \pm 20$ & $28 \pm 12$ \\
\hline Feb & $122 \pm 32$ & $62 \pm 19$ & $91 \pm 126$ & $105 \pm 44$ & $61 \pm 89$ & $23 \pm 13$ \\
\hline Mar & $159 \pm 48$ & $44 \pm 19$ & $91 \pm 126$ & $234 \pm 44$ & $109 \pm 93$ & $0 \pm 11$ \\
\hline Abr & $0 \pm 13$ & $40 \pm 20$ & $0 \pm 126$ & $19 \pm 52$ & $129 \pm 97$ & $0 \pm 9$ \\
\hline May & 0 & $38 \pm 22$ & $91 \pm 126$ & 0 & 0 & $0 \pm 4$ \\
\hline Jun & $62 \pm 64$ & $16 \pm 22$ & $37 \pm 154$ & $71 \pm 90$ & $65 \pm 102$ & $103 \pm 18$ \\
\hline \multicolumn{7}{|c|}{ Tabla 4: Continuación. } \\
\hline Mes & XJ3-401234 & XJ3-400332 & XJ3-401432 & XJ3-401132 & XJ3-401231 & XJ3-200918 \\
\hline Ene & $34 \pm 22$ & $50 \pm 22$ & $16 \pm 14$ & $27 \pm 11$ & $43 \pm 07$ & $14 \pm 35$ \\
\hline Feb & $6 \pm 22$ & $41 \pm 29$ & $4 \pm 17$ & $0 \pm 15$ & $21 \pm 11$ & $22 \pm 35$ \\
\hline Mar & $19 \pm 26$ & $0 \pm 26$ & $42 \pm 19$ & $12 \pm 19$ & $25 \pm 11$ & $12 \pm 35$ \\
\hline Abr & $29 \pm 27$ & $0 \pm 12$ & $35 \pm 20$ & $0 \pm 22$ & $62 \pm 11$ & $12 \pm 35$ \\
\hline May & $32 \pm 27$ & $0 \pm 31$ & $0 \pm 17$ & $31 \pm 22$ & $45 \pm 13$ & $0 \pm 7$ \\
\hline Jun & $79 \pm 27$ & $4 \pm 39$ & $37 \pm 20$ & $0 \pm 22$ & $39 \pm 15$ & $22 \pm 35$ \\
\hline
\end{tabular}

Tabla 5: Características de las unidades a almacenar

\begin{tabular}{|l|c|c|c|c|}
\hline \multicolumn{1}{|c|}{ Descripción } & Alto cm & Ancho cm & Profundidad cm & Peso kg \\
\hline Tarima & 13.5 & 101.5 & 120.0 & 30.0 \\
\hline Tarima 36 & 104.0 & 101.5 & 120.0 & 630.0 \\
\hline Tarima 48 & 138.0 & 101.5 & 120.0 & 840.0 \\
\hline Caja (4 Gal) & 20.5 & 35.0 & 35.0 & 17.0 \\
\hline Cubeta & 34.5 & 30.0 & 30.0 & 17.5 \\
\hline Galón & 19.5 & 16.5 & 16.5 & 4.0 \\
\hline Litro & 13.0 & 10.8 & 10.8 & 1.0 \\
\hline
\end{tabular}

\section{Estimación de espacio de almacenaje}

La Tabla 6 muestra el análisis producto-tarimas de la totalidad de productos manejados en el almacén para un mes. El resultado fue que se debe de contar con espacio para 101 tarimas si las cubetas -que representan el mayor volumen- se estiban en T-48. Si se estiban en T-36 se requiere espacio para 132 tarimas. Si la mayor demanda de espacio (132 tarimas) se divide en cuatro semanas, se tiene una demanda de espacio semanal de 33 tarimas. La actual capacidad de almacenaje de tarimas en estantes es de 48, por lo que se tiene una holgura de 15 tarimas, lo que representa 3 estantes vacíos.

Tabla 6: Estimado de unidades por análisis directo

\begin{tabular}{|l|c|l|c|c|}
\hline Presentación & Venta en mes & \multicolumn{1}{|c|}{ Unidades de envío } & Cantidad & Espacio en $\mathrm{m}^{3}$ \\
\hline \multirow{2}{*}{ Cubeta } & \multirow{2}{*}{4,522} & Tarima 36 & 126 & 161.30 \\
\cline { 3 - 5 } & & Tarima 48 & 95 & 161.50 \\
\hline \multirow{2}{*}{ Galón } & \multirow{2}{*}{1,216} & Caja 4 piezas & 304 & 7.65 \\
\cline { 3 - 5 } & & Tarima 54 cajas & 6 & 10.32 \\
\hline Litro & 42 & Caja 6 piezas & 7 & 0.132 \\
\hline
\end{tabular}

El espacio cúbico total indicado en la Tabla 6 es considerando únicamente el volumen exacto de las tarimas, sin espacios adicionales para maniobrar las tarimas. La dimensión frontal de los estantes actuales de la empresa tienen $226 \mathrm{~cm}$ y el ancho de las tarimas es de $102 \mathrm{~cm}$. El espacio empleado por dos tarimas es de $204 \mathrm{~cm}$, por lo que se tiene una holgura de $22 \mathrm{~cm}$ para realizar maniobras. Este espacio es adecuado para realizar maniobras sin afectar la carga, considerando que Tejero (2008) indica que debe de existir una separación de $5 \mathrm{~cm}$ de cada tarima con los largueros traseros de las estanterías, y $10 \mathrm{~cm}$ de separación lateral. 
Una buena administración reduce la cantidad de elementos requeridos en los almacenes e incrementa los niveles de cumplimiento de los pedidos de clientes (Arango et al., 2011). En la Tabla 7 se observa que únicamente en el mes de noviembre la estantería actual no tiene capacidad para las necesidades de almacenaje. En el resto de los meses se tiene holgura de almacenaje. Sobre este análisis, se determinó realizar una distribución de productos programada con los concesionarios para evitar saturar el almacén. De esta forma se evitarían los costos asociados a cambios en la distribución de la planta, adquisición de estanterías (\$USD 2000), equipo de manejo de materiales (\$USD 7500), costos de cuidado, costos de pedidos especiales, entre otros. Las decisiones estratégicas involucran evaluar la pertinencia de invertir recursos financieros en actividades para innovar, con la finalidad de entregar a la empresa un real apoyo para hacerla más competitiva (Espinosa et al., 2011)

Tabla 7: Proyección del espacio en almacén

\begin{tabular}{|l|r|r|r|r|r|r|r|r|r|r|r|r|}
\hline Descripción & Ene & Feb & Mar & Abr & May & Jun & Jul & Ago & Sep & Oct & Nov & Dic \\
\hline Cubetas (unidades) & 4522 & 3382 & 4465 & 4679 & 2774 & 4706 & 5738 & 4937 & 2467 & 4871 & 12084 & 6153 \\
\hline Tarima 36 & 126 & 94 & 125 & 130 & 78 & 131 & 160 & 138 & 69 & 136 & 336 & 171 \\
\hline Galones (unidades) & 1216 & 1228 & 1184 & 1792 & 1028 & 1632 & 2292 & 1512 & 1220 & 1988 & 3256 & 2588 \\
\hline Tarima 36 & 6 & 6 & 5 & 8 & 5 & 8 & 11 & 7 & 6 & 9 & 15 & 12 \\
\hline Litros (unidades) & 42 & 6 & 30 & 6 & 18 & 12 & 18 & 6 & 30 & 30 & 12 & 42 \\
\hline Cajas 6 & 7 & 1 & 5 & 1 & 3 & 2 & 3 & 1 & 5 & 5 & 2 & 7 \\
\hline Total mes Tarima 36 & 132 & 100 & 130 & 138 & 83 & 139 & 171 & 145 & 75 & 145 & 351 & 183 \\
\hline $\begin{array}{l}\text { Total semanal } \\
\text { Tarima 36 }\end{array}$ & 33 & 25 & 33 & 35 & 21 & 35 & 43 & 37 & 19 & 37 & 88 & 46 \\
\hline Holgura en almacén & 15 & 23 & 15 & 13 & 27 & 13 & 5 & 11 & 29 & 11 & -40 & 2 \\
\hline
\end{tabular}

\section{Determinación de niveles de inventarios}

En total, 21 productos obtuvieron errores menores a $20 \%$, el resto presentaron errores superiores debido a que son productos con alta estacionalidad. De Diego (2015) señala que es complicado pronosticar los productos cuya demanda es intermitente debido a un bajo volumen general y alta incertidumbre respecto a la cantidad y el momento en que se presentará el nivel de demanda. Por su parte, Ballou (2004) indica que cuando existe una tendencia importante o un patrón estacional significativo en la información, el retraso inherente de pronóstico de ajuste exponencial puede arrojar errores inaceptables de pronóstico.

\section{CONCLUSIONES}

Para lograr una mayor eficiencia en las operaciones empresariales no se requieren aplicar técnicas de análisis muy sofisticadas. Una adecuada selección y combinación de herramientas simples -como lluvias de ideas, Estratificación, Diagramas Causa-Efecto, clasificación ABC, entre otras- puede dar resultados satisfactorios a un costo relativamente bajo.

De acuerdo a los resultados obtenidos, la empresa cuenta con los recursos necesarios para llevar a cabo una adecuada operación logística. Esta brindará soluciones y aporte valor a la empresa sin necesidad de invertir más recursos. Se determinó que una adecuada planificación maximiza el espacio en cada localidad y bahía dentro del almacén.

Con la utilización del software Oracle CrystalBal| ${ }^{\circledR}$ se logró construir las políticas de inventario adecuadas. Por medio de mínimos, máximos y puntos de reorden se mantiene un resurtido dinámico para los 63 códigos analizados. No obstante, las proyecciones de ventas de productos estacionales presentan un mayor error por la variación en las ventas.

Para mejorar la confiabilidad de las proyecciones, si se cuenta con pocos datos, se sugiere considerar el método SARIMA. EI MAPE es sensible y no se recomienda emplearlo cuando se cuenta con pocos datos.

\section{REFERENCIAS}

Andrade, J., Olivares, A., y M. Robles, La planeación y control del costo de producción en las pequeñas empresas manufactureras, como herramientas que faciliten el cumplir tiempos de entrega del producto terminado, http://www.epistemus.uson.mx/revistas/articulos/17-11EMPRESAS_MANUFACTURERAS.pdf, ISSN: 2007-8196, Epistemus: 17, 75-80 (2014) 
Arango, M.D., Zapata, J.A., y W.A.Jaimes, Aplicación del modelo de inventario manejado por el vendedor en una empresa del sector alimentario colombiano, http://www.redalyc.org/articulo.oa?id=149222633003, ISSN: 1794-1237, Revista EIA: 15, 21-32 (2011)

Ballou, R.H., Logística: administración de la cadena de suministro, $5^{\text {ta }}$ edición, pp 299., Pearson Educación, Estado de México, México (2004)

Carlo, H.J., y G.E. Giraldo, Toward perpetually organized unit-load warehouses, doi: 10.1016/j.cie.2012.06.012, Comput. Ind. Eng. 63, 1003-1012 (2012)

De Diego A., UF0929 - Gestión de pedidos y stock. pp 124., Ediciones Paraninfo, S.A.,España (2015)

Espinosa, F., Salinas, G. y P. Leiva, Jerarquización del Reemplazo de Equipos Productivos de Acuerdo a su Nivel de Cumplimiento de los Objetivos de la Empresa, doi: 10.4067/S0718-07642011000400011, Inf. tecnol. [online], 22 (4), 97-106 (2011)

Gutiérrez V., y C. Vidal, Modelos de Gestión de Inventarios en Cadenas de Abastecimiento: Revisión de la Literatura, http://www.scielo.org.co/scielo.php?pid=S0120-62302008000100012\&script=sci_arttext, ISSN 0120-6230, Rev. Fac. Ing. Univ. Antioquia, 43, 134-149 (2008)

Hatefi S., y S. Torabi, A Common Weight Linear Optimization Approach for Multicriteria ABC Inventory Classification, doi: 10.1155/2015/645746, Advances in Decision Sciences, ID 645746, 11, (2015)

Kumar, S., y L. Vanajakshi, Short-term traffic flow prediction using seasonal ARIMA model with limited input data, doi: 10.1007/s12544-015-0170-8, Eur. Transp. Res. Rev. 7(21), 1-9 (2015)

Li, J., Moghaddam, M., y S.Y. Nof, Dynamic storage assignment with product affinity and ABC classificationa case study, doi:10.1007/s00170-015-7806-7, Int. J. Adv. Manuf. Technol. 1-16 (2015).

Murthy, A., y S. Gurusamy, Cost Accounting, $2^{\text {da }}$ edición, pp 1064, Tata McGraw-Hill Education, New Delhi, India (2009)

Pan, S., Nigrelli, M., Ballot, E., Sarraj, R., y Y. Yang, Perspectives of inventory control models in the Physical Internet: A simulation study, doi: 10.1016/j.cie.2014.11.027, Comput. Ind. Eng., Intelligent Enterprise Systems 84, 122-132 (2015)

Pazour, J.A., y H.J. Carlo, Warehouse reshuffling: Insights and optimization, doi:10.1016/j.tre.2014.11.002, Transp. Res. Part E Logist. Transp. Rev, 73, 207-226 (2015)

Periasamy P., Financial Management, 2da edición, 22.18-22.21, Tata McGraw-Hill Education, New Delhi, India (2009)

Sarpong, S., Modeling and Forecasting Maternal Mortality; an Application of ARIMA Models, http://www.ijastnet.com/journals/Vol_3_No_1_January_2013/3.pdf, ISSN 2221-0997, Int. J. Appl. Sci. Technol.: 3(1), 19-28 (2013)

Tejero, J.A., Almacenes: Análisis, diseño y organización, 174., ESIC Editorial, Madrid, España (2008)

Téllez, J., Rodríguez, A., y Á. Fajardo, Contaminación por Monóxido de Carbono: un Problema de Salud Ambiental, doi: 0.7705/biomedica.v35i0.2449, Carbon Monoxide Contam. Environ. Health Probl. Engl. 8(1), 108-117 (2006)

Trujillo, M., Zapata, A. y W. Sarache, Metodología Integral para el Mejoramiento de la Calidad Mediante la Reducción de la Variabilidad Funcional: Un Caso de Estudio, doi:10.4067/S0718-07642015000600021, Inf. tecnol. [online], 26 (6), 211-221 (2015)

Wu, Z., Zhai, X., y Z. Liu, The inventory billboard effect on the lead-time decision, DOI: 10.1016/j.ijpe.2015.09.008, Int. J. Prod. Econ. 170 (Part A), 45-53 (2015) 